\title{
Multiphoton microscopy of engineered dermal substitutes: assessment of 3-D collagen matrix remodeling induced by fibroblast contraction
}

\author{
Ana-Maria Pena \\ Dominique Fagot \\ Christian Olive \\ Jean-François Michelet \\ Jean-Baptiste Galey \\ Frédéric Leroy \\ L'Oréal Research and Innovation \\ 1 Avenue Eugene Schueller BP22 \\ 93601 Aulnay Sous Bois, France
}

\author{
Emmanuel Beaurepaire \\ Jean-Louis Martin \\ CNRS \\ Ecole Polytechnique \\ Laboratoire d'Optique et Biosciences \\ 91128 Palaiseau, France \\ and \\ INSERM \\ U696 \\ 91128 Palaiseau, France
}

\section{Anne Colonna}

L'Oréal Research and Innovation 1 Avenue Eugene Schueller BP22

93601 Aulnay Sous Bois, France

\author{
Marie-Claire Schanne-Klein \\ CNRS \\ Ecole Polytechnique \\ Laboratoire d'Optique et Biosciences \\ 91128 Palaiseau, France \\ and \\ INSERM \\ U696 \\ 91128 Palaiseau, France
}

\begin{abstract}
Dermal fibroblasts are responsible for the generation of mechanical forces within their surrounding extracellular matrix and can be potentially targeted by anti-aging ingredients. Investigation of the modulation of fibroblast contraction by these ingredients requires the implementation of three-dimensional in situ imaging methodologies. We use multiphoton microscopy to visualize unstained engineered dermal tissue by combining second-harmonic generation that reveals specifically fibrillar collagen and two-photon excited fluorescence from endogenous cellular chromophores. We study the fibroblastinduced reorganization of the collagen matrix and quantitatively evaluate the effect of Y-27632, a RhoA-kinase inhibitor, on dermal substitute contraction. We observe that collagen fibrils rearrange around fibroblasts with increasing density in control samples, whereas collagen fibrils show no remodeling in the samples containing the RhoA-kinase inhibitor. Moreover, we show that the inhibitory effects are reversible. Our study demonstrates the relevance of multiphoton microscopy to visualize three-dimensional remodeling of the extracellular matrix induced by fibroblast contraction or other processes. (C) 2010 Society of Photo-Optical Instrumentation Engineers. [DOI: 10.1117/1.3503411]
\end{abstract}

Keywords: collagen; second-harmonic generation; multiphoton microscopy; threedimensional imaging; dermal substitutes; fibroblasts.

Paper 10308PR received Jun. 4, 2010; revised manuscript received Jul. 23, 2010; accepted for publication Aug. 30, 2010; published online Oct. 29, 2010.

\section{Introduction}

Dermal fibroblasts are a dynamic population of cells that play an important role in extracellular matrix synthesis and remodeling. Their contractile properties influence the morphology and the mechanical properties of the skin. In skin wound healing, fibroblasts exert mechanical forces within their surrounding extracellular matrix that lead, for instance, to wound contraction. ${ }^{1}$ In this case, the applied forces are large enough to cause scars and even major body deformation if their contraction persists after wound closure. ${ }^{2}$ Dermal fibroblast contraction may thus participate in skin aging that is characterized by the formation of wrinkles.

Address all correspondence to: Ana-Maria Pena, L'Oréal Research and Innovation, 1 Avenue Eugene Schueller BP22, 93601 Aulnay Sous Bois, France. Tel: 33-1-48-68-98-27; E-mail: ampena@rd.loreal.com
Bioengineered dermal skin equivalent ${ }^{3,4}$ made up of fibroblasts in a collagen matrix offer a means to modulate fibroblast contraction in a three-dimensional (3-D) environment and thus to investigate molecules that could act as potent antiaging ingredients. The characterization of the 3-D remodeling of the extracellular matrix induced by fibroblast contraction or other processes in skin equivalents requires the development of in situ imaging methodologies. Contraction assays have been reported that aimed at assessing the effect of biochemical and mechanical parameters on matrix remodeling. ${ }^{2}$ Coupled with 2-D mechanics analysis based on differential interference contrast images of collagen matrix remodeling around fibroblasts, ${ }^{5}$ these methods can provide information about the contraction forces applied by fibroblasts to the surrounding matrix and could be used as a tool to investigate the

1083-3668/2010/15(5)/056018/7/\$25.00 @ 2010 SPIE 
effects of different molecules on the fibroblast cells. However, these conventional imaging methods do not enable 3-D visualization of matrix remodeling in thick samples. Confocal reflection microscopy ${ }^{6}$ and optical coherence tomography $(\mathrm{OCT})^{7}$ can provide noninvasive 3 -D visualization of bioengineered dermal skin equivalents but lack specificity, as their contrast mechanism relies on spatial variations of refractive indices. Three-dimensional interactions between cells and collagen fibrils cannot therefore be studied using this type of contrast mechanism. For that, exogenous fluorescent markers or fluorescent fusion proteins are used to specifically detect the cells or to mark their surrounding environment. In this context, it is crucial to develop highly specific 3-D in situ imaging methodologies that enable simultaneous monitoring of the 3-D collagen network and of the fibroblast distribution in unstained samples. Multiphoton microscopy offers this 3-D multimodal imaging capability, as it provides structural and biochemical information about unstained samples that is not accessible using other noninvasive methods.

In the present study, we use multiphoton microscopy to evaluate the effect of fibroblast contraction inhibitors in a fibroblast-populated fibrillar collagen gel. Multiphoton microscopy indeed presents unique advantages for the visualization of unstained collagenous tissues. It provides intrinsic 3-D resolution that is robust in scattering biological tissues such as collagen matrices and improves depth imaging with submicrometer resolution. Most importantly, this technique presents several modes of contrast that enable simultaneous visualization of different tissue components. The main multiphoton modalities are two-photon excited fluorescence (2PEF) and second-harmonic generation $(\mathrm{SHG}){ }^{8-18}$ They can be excited by the same laser source and detected separately by taking advantage of their spectral difference: $2 \mathrm{PEF}$ signals are indeed red-shifted compared to SHG signals that appear at exactly twice the excitation frequency (half the excitation wavelength). Moreover, they can be used in unstained intact tissues by taking advantage of endogenous sources of contrasts. 2 PEF signals are emitted by endogenous chromophores such as NAD $(\mathrm{P}) \mathrm{H}$, flavins, keratin, or elastin. ${ }^{11}$ They can be exploited for the visualization of fibroblasts by taking advantage of NAD(P)H fluorescence on excitation around $730 \mathrm{~nm}$. SHG signals are specific for dense and ordered macromolecular structures such as fibrillar collagen and can be used to image the collagenous network in 3-D matrices. ${ }^{10,19-24}$ Simultaneous recording of $2 \mathrm{PEF}$ signals from cellular chromophores and SHG signals from fibrillar collagen therefore enables in situ multimodal 3-D imaging of engineered tissues. ${ }^{10,25}$

The aim of this study is to implement a sensitive and minimally invasive 3-D technique for monitoring contraction assays in fibroblast-populated collagen gels. For that purpose, we combine SHG and 2PEF microscopies to visualize collagen remodeling around fibroblasts in unstained samples, and we develop a quantitative analysis of our 3-D multimodal images to measure the collagen density around fibroblasts. We apply this technique to Y-27632, a RhoA-kinase inhibitor, ${ }^{26}$ which is known to impede the accumulation of collagen fibrils around fibroblasts, and we precisely characterize its effect in a 3 -D environment. Indeed, the contraction properties of fibroblasts are regulated principally by the activation of the contractile proteins myosin and actin. For contraction to occur, myosin light chain kinase (MLC kinase) must phosphorylate the light chain of myosin, enabling the molecular interaction of myosin with actin. The process of relaxation is initiated by myosin phosphatase, whose activation is down-regulated by RhoA-kinase. Y-27632 is known to block the RhoA-kinase activity, so that the myosin phosphatase is activated and relaxation occurs. ${ }^{6,27,28}$

In the following, we first introduce our multiphoton setup and the protocols used for modulating fibroblast contraction in dermal substitutes. We then present the experimental results and the quantitative analysis of the multiphoton images to assess fibroblast contraction efficiency. We then draw conclusions about the sensitivity and reliability of this new methodology.

\section{Materials and Methods}

\subsection{Sample Preparation}

Collagen solutions with dermal normal human fibroblasts were prepared as previously described. ${ }^{3,4}$ Briefly, $4.6 \mathrm{ml}$ MEM $2 \times, 0.9 \mathrm{ml}$ fetal calf serum, $0.39 \mathrm{ml} \mathrm{MEM}, 0.5 \mathrm{ml}$ $\mathrm{NaOH} 0.1 \mathrm{~N}, 2.6 \mathrm{ml}$ collagen type I and $1 \mathrm{ml}$ fibroblast suspension $\left(1.4 \times 10^{5}\right.$ cells $\left./ \mathrm{ml}\right)$ were successively added in centrifuge tubes in the presence or absence of $10 \mu \mathrm{M}$ Y-27632. These collagen solutions $(2 \mathrm{ml}$ at $1 \mathrm{mg} / \mathrm{ml}$ final concentration and $3 \times 10^{4}$ fibroblasts) were seeded in individual wells of a 12-well culture dish. The solutions were incubated for 3 days in a cell culture medium $(0.5 \mathrm{ml} \mathrm{MEM}$ with $0.9 \mathrm{ml}$ of fetal calf serum) at $37{ }^{\circ} \mathrm{C}$ and $5 \% \mathrm{CO}_{2}$. Fibrillar gels were obtained after the first two hours through matrix polymerization and fibrils formation, and they attached to the well plate walls. They were kept attached to the walls during the 3 day incubation period so that this mechanical constraint impeded any matrix contraction before $T 0$.

At $T 0$, contraction of the collagenous lattices was induced by mechanical detachment of the gel from the well plate walls [see the schematized assay in Fig. 1(a)]. Two samples per condition were studied at each of three different times. Two control samples and two treated samples were imaged immediately after detachment. The other samples were maintained in the incubator in the same conditions as before $\left(37^{\circ} \mathrm{C}, 5 \%\right.$ $\left.\mathrm{CO}_{2}\right)$. After $24 \mathrm{~h}(T+24 \mathrm{~h})$, two other control samples and two other treated samples were imaged. At the same time, the culture medium of the remaining treated samples with Y-27632 was removed and replaced by a control medium without inhibitor. Last, after $48 \mathrm{~h}(T+48 \mathrm{~h})$, the last two control samples and two treated samples were imaged.

\subsection{Multiphoton Setup}

Multiphoton imaging was performed using a custom-built upright laser scanning microscope, as previously described. ${ }^{16-18}$ SHG and 2PEF were simultaneously excited by a femtosecond titanium-sapphire laser adjusted to $730 \mathrm{~nm}$, with typically $60-\mathrm{mW}$ power at the sample (Tsunami, SpectraPhysics). These signals were dispatched to two photoncounting epidetection nondescanned channels using a dichroic mirror (390DRLP, Omega Filters, Brattleboro, Vermont) and appropriate spectral filters: GG400 and E700SP-2P (Chroma, Rockingham, Vermont) for the 2PEF channel; FI01-355/40 (Semrock, Rochester, New York) and BG39 for the SHG channel. We used circular polarization in order to minimize 


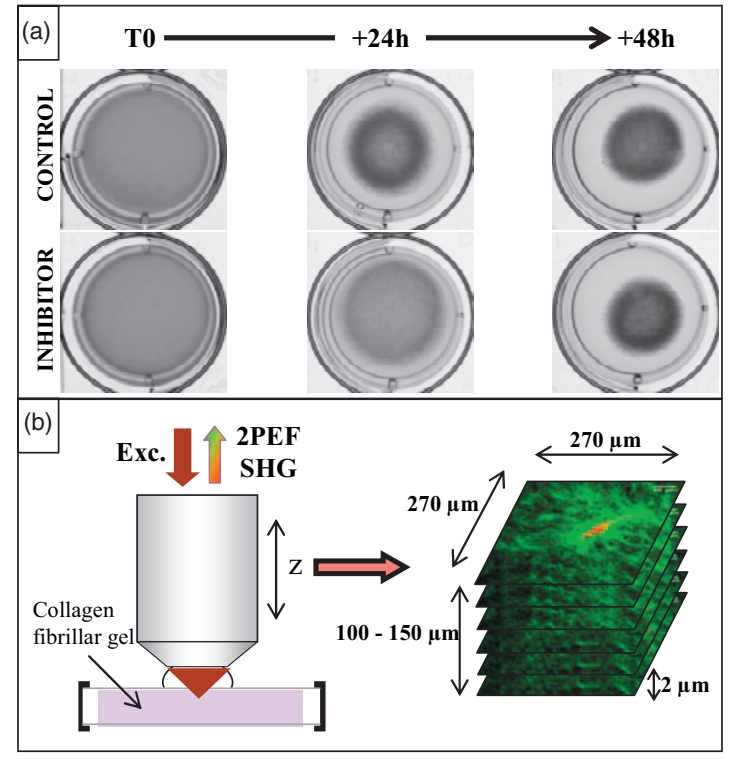

Fig. 1 Imaging configuration and contraction assay. (a) Protocol for the evaluation of fibroblast-induced collagen matrix reorganization in dermal substitutes. Contraction of the collagenous lattices is induced at $T 0$ by mechanical detachment of the gel from the well-plate edges. Multiphoton imaging is performed at TO (before contraction occurs), after $24 \mathrm{~h}(T+24 \mathrm{~h})$ and after $48 \mathrm{~h}(T+48 \mathrm{~h})$ of contraction. (b) Scheme of the imaging configuration showing epidetection of $2 \mathrm{PEF}$ and SHG signals. Objective: 20×, 0.9 NA. Excitation: $60 \mathrm{~mW}$ at $730 \mathrm{~nm}$.

orientation effects in the image plane. Samples were maintained between two cover slides and imaged without any staining by use of a $20 \times, 0.95-N A$ objective lens (Olympus, Japan) with approximately $0.4 \mu \mathrm{m}$ (lateral) $\times 1.6 \mu \mathrm{m}$ (axial) resolutions near the sample surface. No photodamage was observed in these experimental conditions. We recorded typically four $270 \times 270 \times 100$ to $150 \mu \mathrm{m}^{3} z$-stacks in every sample, with $2-\mu \mathrm{m} z$-step and $0.4-\mu \mathrm{m}$ pixel size [see Fig. 1(b)]. The pixel rate was $100 \mathrm{kHz}$, and the acquisition time was $7 \mathrm{~s}$ per image $(681 \times 681$ pixels $)$. Two-dimensional images were combined using ImageJ (W. Rasband, National Institutes of Health), and 3-D reconstructions were performed with Amira (Mercury Computed Systems).

\subsection{Quantitative Analysis of Collagen Fibrils Remodeling}

SHG and 2PEF images were combined using ImageJ to obtain multimodal $z$-stacks and to assess collagen remodeling around fibroblasts. Fibroblasts were first retrieved in combined SHG/ $2 \mathrm{PEF} z$-stacks, and 3-D regions of interest (ROIs) of 60 $\times 80 \times 20 \mu \mathrm{m}^{3}$ were defined around the fibroblast body [see Figs. 2(a) and 2(b)]. These ROIs were then processed using macros developed with ImageJ to obtain the volume of the fibroblast body from the 2PEF image $z$-stack and the volume occupied by the collagen fibrillar matrix from the SHG image $z$-stack. For that purpose, we used the following procedure derived from the method we developed for fibrosis scoring ${ }^{16,17}$ to remove the pixels with nonsignificant signal and to enhance the fibrils and fibroblasts contrasts. We performed a Gaussian blur with 1-pixel sigma radius [see Fig.

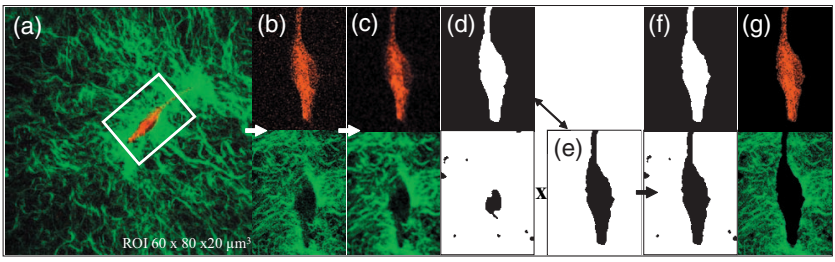

Fig. 2 Video and image processing for quantitative analysis of collagen gel remodeling around fibroblasts. (a) Multiphoton multimodal image from a z-stack acquired in a control fibroblast-populated collagen gel $(T+24 \mathrm{~h})$ : 2PEF signal (red color) reveals the fibroblasts, and SHG signal (green color) reveals the collagen fibrils. The 3-D region of interest ( $\mathrm{ROI}, 60 \times 80 \times 20 \mu \mathrm{m}^{3}$ ) centered on the fibroblast body and used for the quantification procedure is depicted as a white rectangle. The 3-D organization is better seen in the video file. (b) Separated SHG and 2PEF images within the ROI; (c) same after Gaussian blurring (sigma radius $=1$ pixel); (d) SHG and 2PEF masks obtained after application of a threshold; (e) inverted 2PEF mask; (f) 2PEF mask and corrected SHG mask around fibroblasts obtained by multiplication of the inverted fibroblast mask with the collagen mask; (g) corrected 2 PEF and SHG images obtained by multiplication of (b) and ( $f$ ) images. The fibroblast body volume is obtained from the 2PEF mask, and the collagen density is obtained from the corrected SHG mask. (QuickTime, 4.79 MB) [URL: http://dx.doi.org/10.1117/1.3503411.1].

2(c)], and we applied a threshold equal to the maximum background level to obtain a mask [see Fig. 2(d)]. The SHG mask was moreover multiplied by the inverted 2PEF mask [see Fig. 2(e)] to remove SHG signals detected within the fibroblast body because of the moderate axial resolution [see the corrected SHG mask in Fig. 2(f)]. The SHG and 2 PEF raw images were then multiplied by the SHG and 2PEF masks to obtain corrected images [see Fig. 2(f)]. The fibroblast body volume was obtained as the number of voxels of the $2 \mathrm{PEF}$ mask. The collagen volume was obtained as the number of voxels of the corrected SHG mask. We then expressed the collagen fibrils density around fibroblast as the ratio of the collagen volume to the difference between the total ROI volume and the fibroblast body volume. This normalization aimed at comparing the collagen fibrils density around fibroblasts with different sizes.

\subsection{Statistical Analysis}

We analyzed typically 10 fibroblasts (from two different samples) per experimental conditions. To compare control and inhibitor treated samples, we first performed a descriptive analysis using box-and-whiskers plots (see Fig. 3). In order to choose the appropriate test for the analysis of the data, we first verified the hypothesis of normality (histograms, ShapiroWilk algorithm) and of homogeneity of variances (Bartlett's test) that are required for most of the statistical tests. These hypotheses were rejected because some of the data contain outliers (see Fig. 3) and thus deviate from normality. We then transformed the data using logarithmic transformation and found that the normality hypothesis was now accepted for all the samples, but not the hypothesis of homogeneity of variances. Based on these considerations, we chose to use the R-estimators test, ${ }^{29}$ which does not require a normal distribu- 


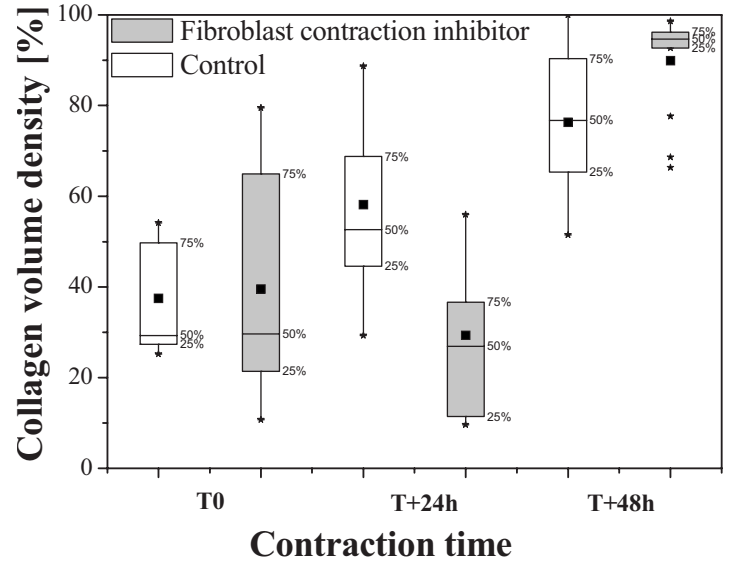

Fig. 3 Descriptive statistical analysis of the quantification of collagen remodeling around fibroblasts. The data are expressed as box plots with fences. The boxes contain $50 \%$ of the data; the intervals between the lower limit of the box and the lower inner fence contain $25 \%$, and vice versa for the other $25 \%$.-indicates the median that divides the population in two groups with equal numbers of data points; $\mathbf{D}$ indicates the position of the mean and * the outliers (atypical data points). This graph shows that the inhibitor-treated sample at $T$ $+48 \mathrm{~h}$ presents outliers and deviates from a normally distributed population (data included in the region delimited by the box and the fences).

tion of the data or the homogeneity of variances. The analyses were performed using SAS software (SAS Institute Inc., Cary, NC). The collagen fibrils density around fibroblasts was expressed as the mean $\pm 95 \%$ confidence intervals of the mean.

\section{Results and Discussion}

Contraction assays treated or not treated with Y-27632, a known fibroblast contraction inhibitor, were conducted using multiphoton microscopy. Our goal is to develop a sensitive and minimally invasive methodology that is able to evaluate the effects of fibroblast contraction inhibitors in a 3-D environment. For that purpose, combined SHG images from fibrillar collagen and $2 \mathrm{PEF}$ images from fibroblasts were acquired upon 730-nm excitation. Typical results are displayed in Figs. 4 and 5 and show the fibroblasts embedded in the fibrillar collagen matrix. Multiphoton microscopy provides unique advantages here. The 3-D and multimodal capabilities (see Fig. 5 and video linked to Fig. 2) enable the visualization of the architecture of the collagenous network around the fibroblasts with high contrast. Moreover, no sample preparation is required, which permits in situ imaging of intact matrices and limits any artifact due to fixation or staining.

At $T 0$, before matrix release, we observed that both control and inhibitor-treated samples exhibited the same characteristic features: the collagen fibrils were straight, and they showed similar densities over all the samples (around fibroblasts or far from fibroblasts). After $24 \mathrm{~h}$ of free contraction, the control and treated samples displayed different matrix organization. In the control samples, we observed an increase of the collagen fibrils density that was larger around the fibroblast body. This observation at the submicrometer scale was consistent with the global matrix contraction that was observed at the macroscopic scale [see Fig. 1(a)]. We attributed this retraction to the rearrangement of existing collagen fibrils, presumably through the application of mechanical forces by fibroblasts.

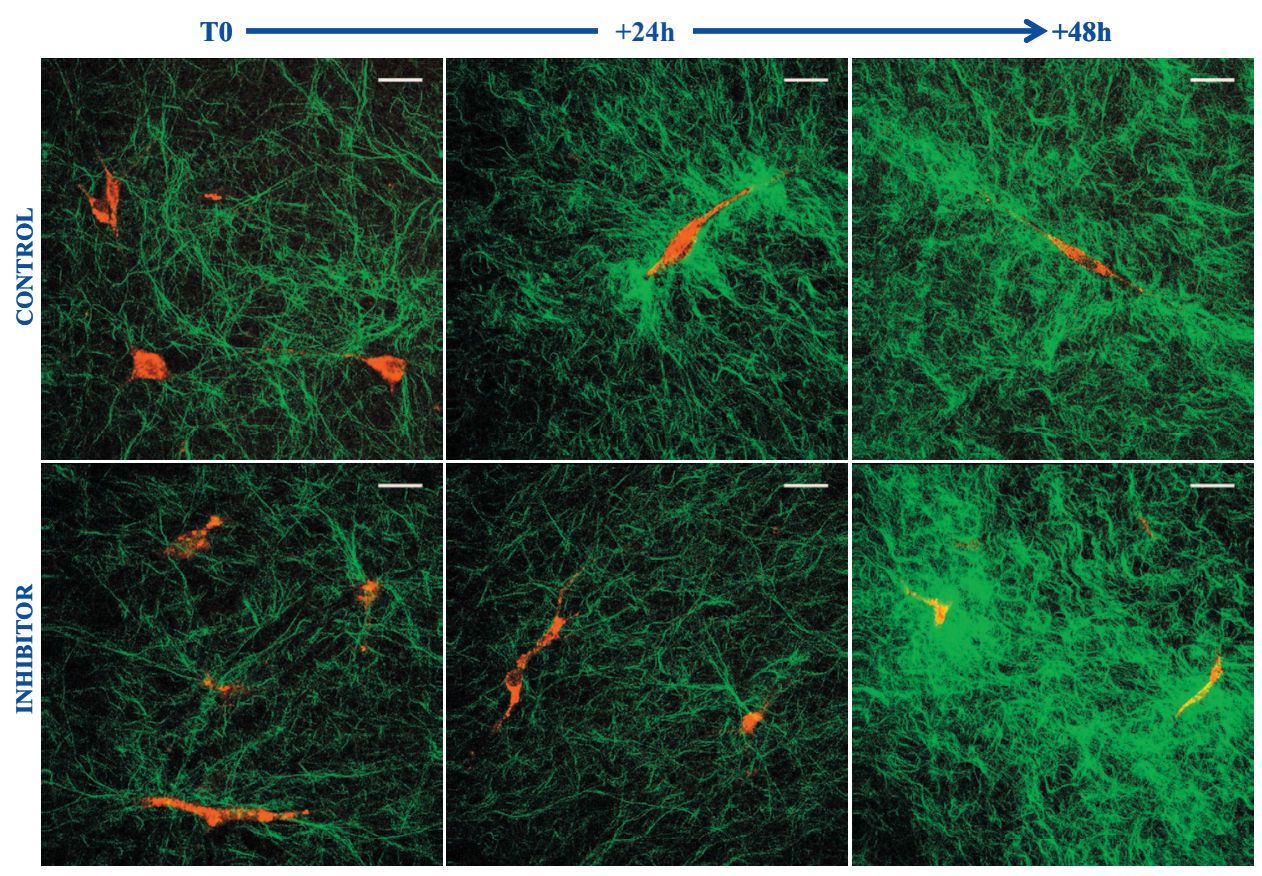

Fig. 4 Multiphoton imaging of collagen matrix remodeling induced by fibroblast contraction. Combined 2PEF (red) and SHG (green) images of fibroblasts within control and Y-27632 treated collagen gels. The images were acquired at $T 0$ - before samples contraction; $T+24 \mathrm{~h}$ and $T$ $+48 \mathrm{~h}-24 \mathrm{~h}$ and $48 \mathrm{~h}$ after free contraction of the samples. At $T+24 \mathrm{~h}$, the fibroblast inhibitor was removed from the culture medium and replaced with a control culture medium in order to assess the reversibility of the inhibitor effect. Scale bar: $30 \mu \mathrm{m}$. Excitation: $60 \mathrm{~mW}$ at $730 \mathrm{~nm}$. Objective: $20 \times, 0.9$ NA. Acquisition time: 6.9 s/image of $681 \times 681$ pixels. 

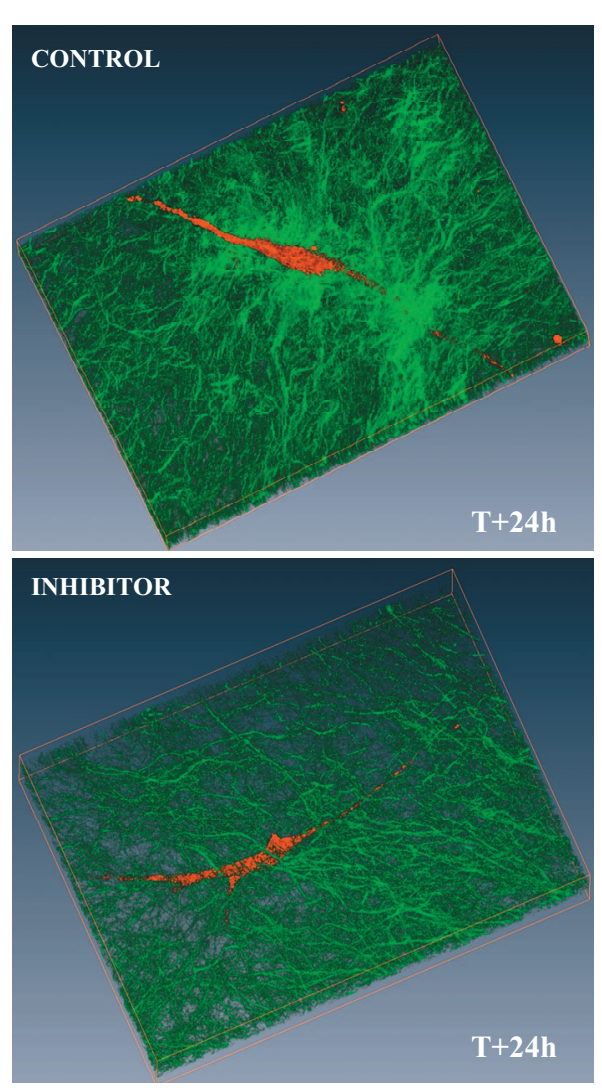

Fig. 5 3-D reconstructed images of collagen organization (SHG, green) around fibroblasts. (2PEF, red) in control and Y-27632-treated collagen gels at $T+24 \mathrm{~h}$ after contraction. Image size: $196 \times 272$ $\times 30 \mu \mathrm{m}^{3}$. Excitation: $60 \mathrm{~mW}$ at $730 \mathrm{~nm}$. Objective: $20 \times, 0.9 \mathrm{NA}$.

The inhibitor treated samples exhibited no remodeling of the fibrillar collagen matrix, no increase of the fibrils density around fibroblasts, and, consistently, no global matrix contraction [see Fig. 1(a)]. This indicated that Y-27632 efficiently blocked the fibroblast-induced matrix contraction. The changes in collagen density around fibroblasts between treated and control samples could also be observed in Fig. 5, which shows 3-D reconstructed images from the multimodal $z$-stacks at $T+24 \mathrm{~h}$. In addition to changes in their density, collagen fibrils also experienced changes in their orientation around the fibroblasts body. Thus, the fibrils became aligned more perpendicular to the fibroblast body (radial alignment) in the treated sample, whereas they were randomly distributed around fibroblasts in the inhibitor-treated samples, in agreement with previous reports. ${ }^{6}$ This type of randomly organized fibrils around fibroblasts was also found in all the samples at $T 0$.

To check whether the inhibitor effects were reversible, the inhibitor was removed from the culture media at $T+24 \mathrm{~h}$, and the samples were kept within the incubator for another $24 \mathrm{~h}$. As shown in Fig. 4, these samples exhibited strong matrix remodeling at $T+48 \mathrm{~h}$, similar to the control samples. This was characterized by an increase of the collagen fibrils density over all the samples, by the presence of curled collagen fibrils, and, consistently, by global matrix contraction [see Fig. 1(a)]. The level of alignment of the collagen fibrils around fibroblasts in these samples was similar to the one in

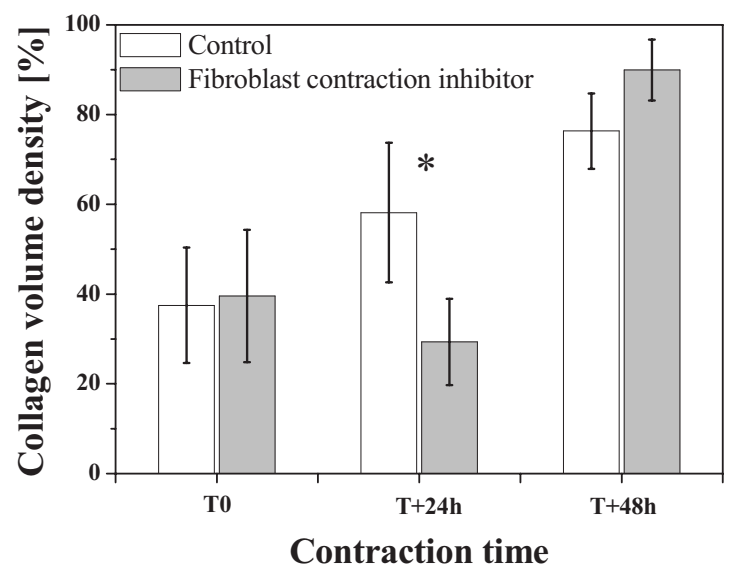

Fig. 6 Quantitative analysis of collagen remodeling around fibroblasts. Mean values of collagen fibrils density around fibroblasts calculated in 3-D ROIs from control and Y-27632-treated samples at T0, $T+24 \mathrm{~h}$, and $T+48 \mathrm{~h}$. Errors bars correspond to $95 \%$ confidence intervals of the mean (see Sec. 2). At T0, there is no difference between control and treated samples, whereas at $T+24 \mathrm{~h}$, the samples show a significant difference $\left({ }^{*} p\right.$-value of 0.0011$)$.

the control samples at $T+24 \mathrm{~h}$. This similar behavior of treated and control samples at $T+48 \mathrm{~h}$ indicated that the effect of the inhibitor was reversible.

In order to more precisely evaluate the inhibitory effects of the RhoA-kinase, we performed a quantitative analysis of our data (see Sec. 2.4). For each sample, we selected 3-D regions of interest centered on the fibroblast body and developed an algorithm that calculated the collagen fibrils density around the fibroblast (see Fig. 2). This procedure allowed for comparison of collagen fibrils densities around fibroblasts with different sizes. The quantification results are shown in Fig. 6 as mean averaged values $\pm 95 \%$ confidence intervals. We obtained no difference between the control and treated samples at $T 0$, a notable difference at $T+24 \mathrm{~h}$, and an increase of the collagen fibrils density around fibroblast with the contraction time in the control samples. To check whether these differences were significant, we performed a descriptive statistics of the data followed by an R-estimators test. ${ }^{29}$ The advantage of using this test compared to other statistical tests is that it can handle data deviating from normality. It requires neither a normal data distribution nor homogeneity of the variances. The results are shown in the Table 1. This statistical analysis confirmed that there was no difference at $T 0$ between the control and treated samples $(p=0.83)$ and no difference between the treated samples at $T 0$ and $T+24 \mathrm{~h} \quad(p=0.37)$, whereas there was a significant difference between the control and treated samples at $T+24 \mathrm{~h}(p=0.0011)$, which confirmed that the inhibitor efficiently blocked the contraction. It also confirmed that the control samples exhibited a significant increase of their collagen fibrils densities with time $(p=0.05$ between $T 0$ and $T+24 \mathrm{~h}, p<0.0001$ between $T 0$ and $T$ $+48 \mathrm{~h}$, and $p=0.008$ between $T+24 \mathrm{~h}$ and $T+48 \mathrm{~h})$. Moreover, we observed a significant increase of the collagen fibrils density in treated samples between $T+24 \mathrm{~h}$ and $T+48 \mathrm{~h}(p$ $<0.0001$ ), which confirmed the reversibility effect of the inhibitor. We observed, however, a difference between the control and treated samples at $T+48 \mathrm{~h}(p=0.033)$ that may be 
Table $1 p$-values obtained from R-estimators tests between the different samples. Significant differences in the collagen fibrils density around fibroblasts are observed between the samples with $p$-values smaller than 0.05, whereas there are no significant differences for the others.

\begin{tabular}{lc}
\hline \multicolumn{1}{c}{ Samples } & p-value \\
\hline Control-T0 versus $T+24 \mathrm{~h}$ & 0.05 \\
Control-T0 versus $T+48 \mathrm{~h}$ & $<0.0001$ \\
Control- $T+24 \mathrm{~h}$ versus $T+48 \mathrm{~h}$ & 0.008 \\
Treated-T0 versus $T+24 \mathrm{~h}$ & 0.37 \\
Treated-T0 versus $T+48 \mathrm{~h}$ & $<0.0001$ \\
Treated-T+24 h versus $T+48 \mathrm{~h}$ & $<0.0001$ \\
$T 0-$ control versus treated & 0.83 \\
$T+24 \mathrm{~h}-$ control versus treated & 0.0011 \\
$T+48 \mathrm{~h}-$ control versus treated & 0.033 \\
\hline
\end{tabular}

related to an adaptive reaction of fibroblasts during the blocking of their contraction by Y-27632. The fibroblasts may then exert stronger forces than the control fibroblasts when the inhibitor was removed and induce more obvious matrix remodeling.

Altogether, this study confirmed that Y-27632 inhibits the matrix contraction by fibroblasts and that this effect is reversible. The methodology we developed enabled precise quantization of this inhibitory effect and may be applied to any compound that modulates matrix remodeling by fibroblast. It illustrates the sensitivity and reproducibility of SHG microscopy, although this micrometer-scale technique is restricted to small regions of the sample (few hundreds of microns). Our results indirectly show that fibroblasts exert strong mechanical forces on the surrounding collagen matrix that lead to collagen remodeling and alignment around the cell body. This work does not, however, provide any measurement of the forces applied by the fibroblasts on the surrounding matrix. For that, traction force assays ${ }^{30}$ could be employed, but such mechanical measurements have been developed in a 2-D environment and require the use of exogenous markers for monitoring the deformations induced by the cells on the environment. It would be challenging to continuously monitor 3-D deformations of the matrix by SHG microscopy and to develop image processing to assess the 3 -D positions and orientations of the collagen fibrils around fibroblasts by generalizing 2-D approaches. ${ }^{5,6,23}$ Such an approach, which is beyond the scope of this paper, would enable new insight into the biomechanics of unstained fibroblast-populated gels by use of SHG microscopy.

\section{Conclusion}

In summary, our study focused on the development of in situ imaging methodologies for the evaluation of molecules that could act as potent anti-aging ingredients by modulating the dermal fibroblasts contraction and remodeling the extracellu- lar matrix. Multimodal multiphoton imaging along with quantitative analysis of the collagen fibrils density around fibroblast unambiguously evidenced the reversible anticontracting activity of the RhoA-kinase inhibitor Y-27632. These results demonstrate the relevance of multiphoton microscopy to visualize matrix remodeling induced by fibroblast contraction and to quantitatively evaluate the effect of active compounds that modulate the fibroblast contractile properties. A perspective is to implement dynamic monitoring of matrix contraction and to map the 3-D deformation of the collagen fibrils around fibroblasts. Last, this new methodology could be easily generalized to the evaluation of other active compounds, including contraction-enhancing compounds by use of suitable dermal substitutes.

\section{Acknowledgments}

We acknowledge administrative support from Laure Lachapelle and Christelle Français and technical support from Xavier Solinas and Jean-Marc Sintès. We thank Thérèse Baldeweck, Charles Gomes, and Mathias Strupler for stimulating discussions.

\section{References}

1. J. M. Sorrell and A. I. Caplan, "Fibroblast heterogeneity: more than skin deep," J. Cell. Sci. 117(5), 667-675 (2004).

2. F. Grinnell, "Fibroblast-collagen-matrix contraction: growth-factor signaling and mechanical loading," Trends Cell Biol. 10, 362-365 (2000).

3. E. Bell, B. Ivarsson, and C. Merrill, "Production of a tissue-like structure by contraction of collagen lattices by human fibroblasts of different proliferative potential in vitro," Proc. Natl. Acad. Sci. U.S.A. 76(3), 1274-1278 (1979).

4. E. Bell, S. Sher, B. Hull, C. Merrill, S. Rosen, A. Chamson, D. Asselineau, L. Dubertret, B. Coulomb, C. Lapiere, B. Nusgens, and Y. Neveux, "The reconstitution of living skin," Investig. Dermatol. 81(s1), 2s-10s (1983).

5. S. Vanni, B. C. Lagerholm, C. Otey, D. L. Taylor, and F. Lanni, "Internet-based image analysis quantifies contractile behavior of individual fibroblasts inside model tissue," Biophys. J. 84(4), 27152727 (2003).

6. A. Kim, N. Lakshman, and W. M. Petroll, "Quantitative assessment of local collagen matrix remodeling in 3-D culture: the role of Rho kinase," Exp. Cell Res. 312(18), 3683-3692 (2006).

7. D. Levitz, M. T. Hinds, S. R. Hanson, and S. L. Jacques, "Characterizing matrix remodeling in collagen gels using optical coherence tomography," in Optical Coherence Tomography and Coherence Domain Optical Methods in Biomedicine XIV, J. A. Izatt et al., Eds., Proc. SPIE 7554(1), 75540T (2010).

8. B. R. Masters, P. T. C. So, and E. Gratton, "Multiphoton excitation fluorescence microscopy and spectroscopy of in vivo human skin," Biophys. J. 72, 2405-2412 (1997).

9. P. J. Campagnola, A. C. Millard, M. Terasaki, P. E. Hoppe, C. J. Malone, and W. A. Mohler, "Three-dimensional high-resolution second-harmonic generation imaging of endogenous structural proteins in biological tissues," Biophys. J. 82, 493-508 (2002).

10. A. Zoumi, A. Yeh, and B. J. Tromberg, "Imaging cells and extracellular matrix in vivo by using second-harmonic generation and twophoton excited fluorescence," Proc. Natl. Acad. Sci. U.S.A. 99(17), 11014-11019 (2002).

11. W. R. Zipfel, R. M. Williams, R. Christie, A. Yu. Nikitin, B. T. Hyman, and W. W. Webb, "Live tissue intrinsic emission microscopy using multiphoton-excited native fluorescence and second harmonic generation," Proc. Natl. Acad. Sci. U.S.A. 100, 7075-7080 (2003).

12. G. Cox, E. Kable, A. Jones, I. Fraser, K. F. Marconi, and M. D. Gorrell, "3-dimensional imaging of collagen using second harmonic generation," J. Struct. Biol. 141, 53-62 (2003).

13. K. König and I. Riemann, "High-resolution multiphoton tomography of human skin with subcellular spatial resolution and picosecond time resolution," J. Biomed. Opt. 8(3), 432-439 (2003). 
14. A.-M. Pena, M. Strupler, T. Boulesteix, G. Godeau, and M.-C. Schanne-Klein, "Spectroscopic analysis of keratin endogenous signal for skin multiphoton microscopy," Opt. Express 13(16), 6268-6274 (2005); erratum: 13(17), 6667 (2005).

15. J. A. Palero, H. S. de Bruijn, A. van der Ploeg-van den Heuvel, H. J. C. M. Sterenborg, and H. C. Gerritsen, "In vivo nonlinear spectral imaging in mouse skin," Opt. Express 14(10), 4395-4402 (2006).

16. A.-M. Pena, A. Fabre, D. Débarre, J. Marchal-Somme, B. Crestani, J.-L. Martin, E. Beaurepaire, and M.-C. Schanne-Klein, "Threedimensional investigation and scoring of extracellular matrix remodeling during lung fibrosis using multiphoton microscopy," Microsc. Res. Tech. 70(2), 162-170 (2007).

17. M. Strupler, A.-M. Pena, M. Hernest, P.-L. Tharaux, J.-L. Martin, E. Beaurepaire, and M.-C. Schanne-Klein, "Second harmonic imaging and scoring of collagen in fibrotic tissues," Opt. Express 15(7), 40544065 (2007).

18. M. Strupler, M. Hernest, C. Fligny, J.-L. Martin, P.-L. Tharaux, and M.-C. Schanne-Klein, "Second harmonic microscopy to quantify renal interstitial fibrosis and arterial remodeling," J. Biomed. Opt. 13, 054041 (2008).

19. P. Stoller, K. M. Reiser, P. M. Celliers, and A. M. Rubenchik, "Polarization-modulated second harmonic generation in collagen," Biophys. J. 82(6), 3330-3342 (2002).

20. N. D. Kirkpatrick, J. B. Hoying, S. K. Botting, J. A. Weiss, and U. Utzinger, "In vitro model for endogenous optical signatures of collagen," J. Biomed. Opt. 11(5), 054021 (2006).

21. C. B. Raub, V. Suresh, T. Krasieva, J. Lyubovitsky, J. D. Mih, A. J. Putnam, B. J. Tromberg, and S. C. George, "Noninvasive assessment of collagen gel microstructure and mechanics using multiphoton microscopy," Biophys. J. 92, 2212-2222 (2007).

22. Y. Sun, H. Y. Tan, S. J. Lin, H. S. Lee, T. Y. Lin, S. H. Jee, T. H. Young, W. Lo, W. L. Chen, and C. Y. Dong, "Imaging tissue engi- neering scaffolds using multiphoton microscopy," Microsc. Res. Tech. 71(2), 140-145 (2008).

23. C. Bayan, J. M. Levitt, E. Miller, D. Kaplan, and I. Georgakoudi, "Fully automated, quantitative, noninvasive assessment of collagen fiber content and organization in thick collagen gels," J. Appl. Phys. 105(10), 102042 (2009).

24. T. Abraham, J. Carthy, and B. McManus, "Collagen matrix remodeling in 3-dimensional cellular space resolved using second harmonic generation and multiphoton excitation fluorescence," J. Struct. Biol. 169, 36-44 (2010).

25. A. M. Pena, C. Olive, J. F. Michelet, J. B. Galey, D. Fagot, F. Leroy, J. L. Martin, A. Colonna, and M. C. Schanne-Klein, "Multiphoton microscopy of engineered dermal substitutes: assessment of 3D collagen matrix remodeling induced by fibroblasts contraction," in Photonic Therapeutics and Diagnostics VI, N. Kollias et al., Eds., Proc. SPIE 7548(1), 754802 (2010).

26. M. Uehata, T. Ishizaki, H. Satoh, T. Ono, T. Kawahara, T. Morishita, H. Tamakawa, K. Yamagami, J. Inui, M. Maekawa, and S. Narumiya, "Calcium sensitization of smooth muscle mediated by a Rhoassociated protein kinase in hypertension," Nature 389(6654), 990994 (1997).

27. R. C. Webb, "Smooth muscle contraction and relaxation," $A d v$. Physiol. Edu. 27(4), 201-206 (2003).

28. A. P. Wheeler and A. J. Ridley, "Why three Rho proteins? RhoA, RhoB, RhoC, and cell motility," Exp. Cell Res. 301(1), 43-49 (2004).

29. J. T. Terpstra and J. W. McKean, "Rank-based analysis of linear models using R," J. Stat. Softw. 14(7), 1-26 (2005).

30. M. L. Lombardi, D. A. Knecht, M. Dembo, and J. Lee, "Traction force microscopy in Dictyostelium reveals distinct roles for myosin II motor and actin-crosslinking activity in polarized cell movement," $J$. Cell. Sci. 120(9), 1624-1634 (2007). 